\title{
Ninety Nine
}

National Cancer Institute

\section{Source}

National Cancer Institute. Ninety Nine. NCI Thesaurus. Code C105787.

A natural number greater than ninety-eight and less than one hundred and the quantity that it denotes. 